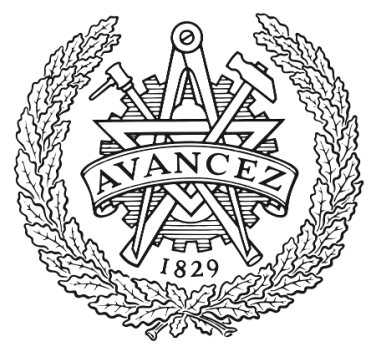

CHALMERS

UNIVERSITY OF TECHNOLOGY

\title{
Microresonator frequency combs for long-haul coherent communications
}

Downloaded from: https://research.chalmers.se, 2023-04-26 11:43 UTC

Citation for the original published paper (version of record):

Fülöp, A., Mazur, M., Lorences Riesgo, A. et al (2018). Microresonator frequency combs for long-haul coherent communications. 2018 International Conference Laser Optics (ICLO): 444-444. http://dx.doi.org/10.1109/LO.2018.8435400

N.B. When citing this work, cite the original published paper. 


\title{
Microresonator frequency combs for long-haul coherent communications
}

\author{
Attila Fülöp ${ }^{1}$, Mikael Mazur ${ }^{1}$, Abel Lorences-Riesgo ${ }^{1,2}$, Tobias A. Eriksson ${ }^{1,3}$, Pei-Hsun Wang ${ }^{4}$, Yi Xuan ${ }^{4,5}$,
} Dan. E. Leaird ${ }^{4}$, Minghao Qi ${ }^{4,5}$, Peter A. Andrekson ${ }^{1}$, Andrew M. Weiner ${ }^{4,5}$, and Victor Torres-Company ${ }^{1}$

${ }^{1}$ Photonics Laboratory, Dept. of Microtechnology and Nanoscience, Chalmers University of Technology, SE-41296 Göteborg, Sweden

${ }^{2}$ Now at IT-Instituto de Telecomunicações, 3810-193 Aveiro, Portugal

${ }^{3}$ Now at National Institute of Information and Communications Technology (NICT), 4-2-1 Nukui-kita, Koganei, Tokyo 184-8795, Japan

${ }^{4}$ School of Electrical and Computer Engineering, Purdue University, West Lafayette, IN 47907-2035, USA

${ }^{5}$ Birck Nanotechnology Center, Purdue University, West Lafayette, IN 47907-2035, USA

\begin{abstract}
Microresonator frequency combs provide a promising platform as multi-wavelength light sources for WDM. The results discussed here show that microresonators can be used in long-haul optical communications systems.
\end{abstract}

\section{Keywords—Coherent communications; Integrated optics.}

\section{INTRODUCTION}

Commercial wavelength-division multiplexed (WDM) communications systems typically employ several free-running lasers. Each laser provides a carrier wave that is modulated and used as a data channel. Demonstrations have shown that there are performance gains when the lasers are replaced by a frequency-stable multi-wavelength light source such as an optical frequency comb. It allows not only decreasing the number of necessary lasers, but its lines' relative stability can be exploited for performance gains through fiber-nonlinearity precompensation [1], guard band minimization [2] or signalprocessing simplification [3]. Of particular interest are microresonator frequency combs using integrated technology [4,5]. Recent experiments have shown impressive aggregate data rates [6] proving the potential of the technology. Here we describe the experiment and the results of long-haul data transmission measurements [7] performed using two normal-dispersion microresonator combs.

\section{COMB-BASED DATA TRANSMISSION}

The two combs shown in Fig. 1 insets, operating in the stable modulation instability regime [8], are used as light sources in a WDM transmission link. The carriers extracted from the comb are modulated with coherent modulation formats (polarization multiplexed (PM) quadrature-phase shift keying (QPSK) and 16-ary quadrature amplitude modulation (16QAM)). The fiber link, consisting of a recirculating loop, allows us to measure bit error ratios (BERs) at regular intervals. Figure 1 displays the BER as a function of link distance. The achieved distances show that microresonatorbased combs are capable of providing light sources for coherent optical WDM systems also in the long-haul regime.

These results will be discussed together with our latest developments, including comb-based transmission using higher order modulation formats [9].

This work has been sponsored by the Swedish Research Council (VR), the European Research Council (ERC), the KA Wallenberg foundation, NSF, DARPA, and AFOSR.
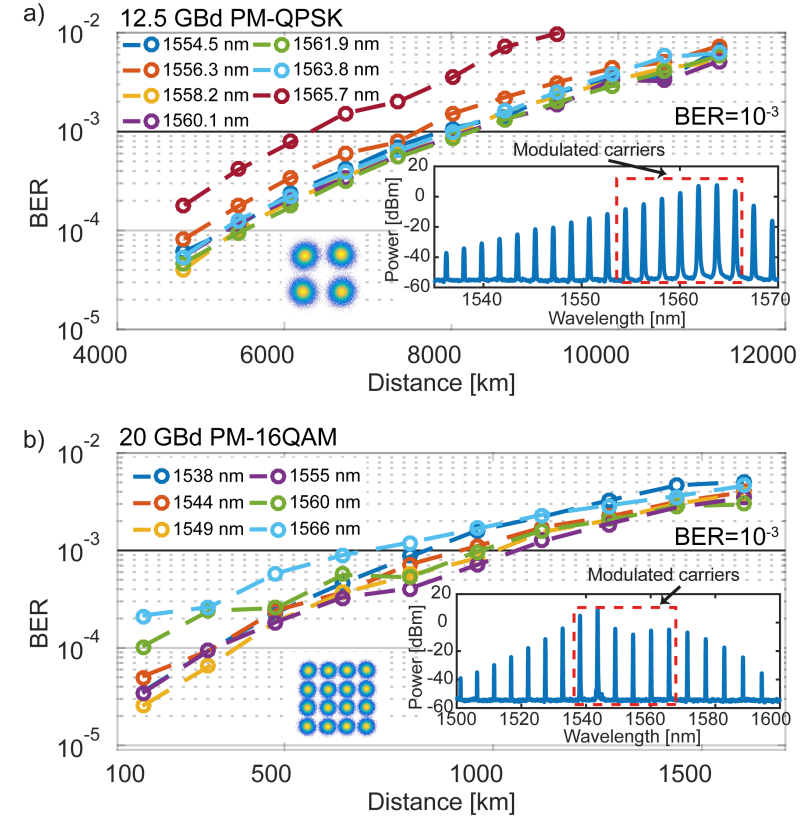

Fig. 1. Long-haul transmission results with the corresponding comb spectra in the insets. a) PM-QPSK transmission over $>6300 \mathrm{~km}$ and b) PM-16QAM transmission over $>700 \mathrm{~km}$ considering a BER-limit of $10^{-3}$. Results from [7].

\section{REFERENCES}

[1] E. Temprana et al., "Overcoming Kerr-induced capacity limit in optical fiber transmission," Science 348, 1445 (2015).

[2] D. S. Millar et al., "Design of a $1 \mathrm{~Tb} / \mathrm{s}$ Superchannel Coherent Receiver," J. Light. Technol. 34, 1453 (2016).

[3] L. Lundberg et al., "Joint Carrier Recovery for DSP Complexity Reduction in Frequency Comb-Based Superchannel Transceivers," Eur. Conf. Opt. Commun. Th1D (2017).

[4] J. S. Levy et al., "CMOS-compatible multiple-wavelength oscillator for on-chip optical interconnects," Nat. Photonics 4, 37 (2010).

[5] J. Pfeifle et al., "Coherent terabit communications with microresonator Kerr frequency combs," Nat. Photonics 8, 375 (2014).

[6] P. Marin-Palomo et al., "Microresonator-based solitons for massively parallel coherent optical communications," Nature 546, 274 (2017).

[7] A. Fülöp et al., "Long-haul coherent communications using microresonator-based frequency combs," Opt. Express 25, 26678 (2017).

[8] S. Coen and M. Erkintalo, "Universal scaling laws of Kerr frequency combs," Opt. Lett. 38, 1790 (2013).

[9] A. Fülöp et al., "High-order coherent communications using mode-locked dark-pulse Kerr combs from microresonators," arXiv 1801.03435 (2018). 